\title{
Avaliação tecnológica em saúde: uso da carboximetilcelulose a $2 \%$ no tratamento das úlceras de perna
}

\author{
Health technology assessment: use of carboxymethylcellulose $2 \%$ in treating leg ulcers \\ Evaluación de tecnologías en salud: uso de la carboximetilcelulosa al $2 \%$ en tratamiento de \\ las úlceras de pierna
}

Luciana Miranda RodriguesI, Beatriz Guitton Renaud Baptista de Oliveira ${ }^{I I}$, Selma Rodrigues de Castilho ${ }^{I I I}$, Débora Omena Futuro ${ }^{I V}$

\begin{abstract}
RESUMO: Estudo de intervenção terapêutica do tipo ensaio clínico não controlado. Objetivou avaliar o custo e a efetividade da carboximetilcelulose $2 \%$ no tratamento das úlceras de perna. Foi aplicado instrumento de avaliação clínica em 20 pacientes de hospital universitário (Niterói-Rio de Janeiro), entre janeiro e outubro de 2010. Após 90 dias de tratamento, os resultados evidenciaram que no grupo A, feridas maiores que $100 \mathrm{~cm}^{2}$, houve redução de $15 \%$ da área lesional. No grupo B, feridas menores que $100 \mathrm{~cm}^{2}$, houve cicatrização total em 33,3\% dos casos. Os custos foram proporcionais à área lesional; quanto maior a área, maior o custo do tratamento. Ao comparar o produto com outros géis do mercado nacional, identificou-se significativa redução do preço da carboximetilcelulose $2 \%(p<0,01)$ produzida na universidade. Conclui-se que a carboximetilcelulose $2 \%$ foi efetiva na redução do tamanho das úlceras, no aumento do tecido de granulação e na diminuição dos custos do tratamento.
\end{abstract}

Palavras-Chave: Enfermagem; úlcera da perna; custos de cuidados de saúde; carboximetilcelulose.

\begin{abstract}
This therapeutic intervention study took the form of an uncontrolled clinical trial. It aimed to evaluate the cost and effectiveness of carboxymethylcellulose $2 \%$ in treating leg ulcers. A clinical evaluation instrument was applied to twenty volunteers at a university hospital in Niteroi, Rio de Janeiro State, between January and October 2010. After ninety days of treatment, the results of group A (lesions larger than 15.5 sq.in.) showed 15\% reduction in lesion area. In group B (wounds smaller than 15.5 sq.in.), healing was total in $33.3 \%$ of cases. The costs were proportional to ulcer area: larger areas entailed higher costs. When this product was compared with other gels on the Brazilian market, the carboxymethylcellulose $2 \%$ produced at the university was observed to yield significant cost reductions $(p<0.01)$. In conclusion, carboxymethylcellulose $2 \%$ was effective in reducing leg ulcers, increasing tissue granulation area and lowering treatment costs.
\end{abstract}

Keywords: Nursing; leg ulcer; health care costs; carboxymethylcellulose.

\begin{abstract}
RESUMEN: Estudio de intervención terapéutica del tipo ensayo clínico no controlado. Objetivo: evaluar el costo y la eficacia de la carboximetilcelulosa $2 \%$ en el tratamiento de úlceras de piernas. Se utilizó un instrumento de evaluación clínica junto a 20 pacientes de un hospital universitario (Niterói, Río de Janeiro), entre enero y octubre de 2010. Después de 90 días de tratamiento, los resultados mostraron que, en el grupo A, de los que tenían heridas más grandes que $100 \mathrm{~cm}^{2}$, hubo una reducción de un $15 \%$ de la zona lesionada. En el grupo B, de personas cuyas heridas eran menores que $100 \mathrm{~cm}^{2}$, la cicatrización completa se observó en el $33,3 \%$ de los casos. Los costos fueron proporcionales a la zona lesionada, cuanto más extensa el área lesionada, más alto el costo del tratamiento. Cuando se comparan el producto con otros geles del mercado nacional, se reveló una reducción significativa del precio de la carboximetilcelulosa $2 \%(p<0,01)$ producida en la universidad. En conclusión, la carboximetilcelulosa $2 \%$ fue eficaz en la reducción del tamaño de las úlceras, en el aumento del tejido de granulación y en la reducción de los costos de tratamiento. Palabras Clave: Enfermería; úlcera de la pierna; costos de la atención en salud; carboximetilcelulosa.
\end{abstract}

\section{INTRODUÇÃO}

O desenvolvimento de novas tecnologias tem proporcionado o aumento da oferta de produtos no mercado. Na área de dermatologia, tem-se encontrado cada vez mais produtos para o tratamento das feridas. O surgimento de curativos mais específicos visa intensificar o processo de cicatrização das lesões e proporcionar ao paciente melhores condições de tratamento e recuperação. Contudo, a grande variedade de produtos, da mesma maneira que possibilita uma melhor adequação e manejo dos diversos tipos de feridas, também demanda um conhecimento especializado na área ${ }^{1,2}$.

${ }^{\mathrm{I}}$ Mestre em Ciências do Cuidado em Saúde pela Escola de Enfermagem Aurora Afonso Costa. Especialista em Enfermagem Dermatológica pela Universidade Gama Filho. Professora Assistente I da Escola de Enfermagem Souza Marques. Enfermeira do Instituto Nacional de Traumatologia e Ortopedia. Rio de Janeiro, Brasil. E-mail: dralumiranda@yahoo.com.br.

IIVice-Coordenadora do Mestrado Acadêmico de Ciências do Cuidado em Saúde e Professora Titular do Departamento de Fundamentos de Enfermagem e Administração da Escola de Enfermagem Aurora Afonso Costa. Niterói, Rio de Janeiro, Brasil. E-mail: beatrizguitton@globo.com.

IIIDoutora em Engenharia Biomédica pela Universidade Federal do Rio de Janeiro. Professora Associada II da Faculdade de Farmácia da Universidade Federal Fluminense. Niterói, Rio de Janeiro, Brasil. E-mail: selmarc@globo.com.

${ }^{\mathrm{IV}}$ Doutora em Química de Produtos Naturais pela Universidade Federal do Rio de Janeiro. Professora Adjunta da Faculdade de Farmácia da Universidade Federal Fluminense. Niterói, Rio de Janeiro, Brasil. E-mail: dfuturo@vm.uff.br. 
Na saúde, novos procedimentos e novas técnicas de tratamento são incorporados pelos diversos profissionais, muitas vezes de forma acelerada e mesmo antes de evidências suficientes que comprovem sua segurança e eficácia. Além disso, estas tecnologias não são substitutivas e sim cumulativas ${ }^{3}$, fazendo com que aumentem os recursos materiais para a assistência ao paciente, elevando, também, o custo dos tratamentos.

A preocupação com o adequado gerenciamento dos custos na área da saúde, em virtude da desproporção entre a demanda das necessidades hospitalares e de saúde e a disponibilidade desses recursos, tem chamado a atenção para um enfoque recente, os custos relacionados aos serviços de saúde.

$\bigcirc$ acentuado desenvolvimento científico e tecnológico, desde a Segunda Guerra Mundial, contribuiu para que o complexo econômico da saúde se constituísse como um dos setores de maior desenvolvimento. Ao mesmo tempo, a saúde dos indivíduos e das populações passou a ser considerada um direito a ser preservado, contribuindo para a expansão dos sistemas de saúde e da medicalização das sociedades ${ }^{4}$.

Diante do exposto, este trabalho teve como objetivo avaliar o custo e a efetividade da carboximetilcelulose (CMC) 2\% no tratamento das úlceras de perna.

\section{Referencial TeóRICO}

As úlceras de perna são consideradas um importante problema de saúde pública em todo o mundo, sendo responsáveis por significativo impacto econômico, tanto no âmbito governamental, pelos elevados gastos com o tratamento, quanto na esfera pessoal, pelas limitações físicas e sociais do paciente.

A presença da lesão, a dificuldade de cicatrização, a dor permanente e as limitações físicas, além dos diversos problemas de ordem psicossocial, como o isolamento, levam o paciente à perda da autoestima e ao afastamento do trabalho ${ }^{5-7}$.

As principais causas das úlceras crônicas de membros inferiores são as doenças venosas; 60 a $80 \%$ das úlceras de perna tem componente venoso ${ }^{8}$. Pesquisas apontam que $20 \%$ de todos os diabéticos desenvolvem úlceras de membros inferiores em algum momento da vida e $25 \%$ de todas as internações que acontecem com pacientes portadores de diabetes são por acometimento nos membros inferiores ${ }^{9}$. $O$ pé diabético clássico é provocado principalmente pela neuropatia distal. Clinicamente, são úlceras que se localizam em áreas de pressão, tendo como halo uma área de ceratose ${ }^{10}$. Suas características principais são a ausência de sensibilidade e bordos circulares de localizações mais frequentes nas superfícies plantares.

Apesar de poucos estudos epidemiológicos sobre úlceras de perna, sabe-se que elas são muito frequentes na prática médica e absorvem grande quantidade de recursos da área de saúde destinada a seu manejo ${ }^{11}$. $\mathrm{O}$ tratamento das úlceras de perna é bastante oneroso para as instituições de saúde, pacientes e familiares, uma vez que a lesão tem caráter crônico e recidivante ${ }^{12}$. Cerca de $30 \%$ das úlceras cicatrizadas recorrem no primeiro ano. E, de acordo com o aumento da expectativa de vida da população mundial, sua frequência vem aumentando, pois a prevalência aumenta com a idade, sendo superior a $4 \%$ em pessoas a partir dos 65 anos $^{6,11}$.

No tratamento tópico destas lesões, tem-se considerado os avanços tecnológicos na área de curativo, com a oferta de produtos que, evidentemente, aceleram a cicatrização das feridas e facilitam em muito a vida do paciente. Contudo, alguns fatores são importantes neste aspecto, entre eles: os recursos financeiros do paciente e/ou da unidade de saúde, a necessidade de continuidade da utilização do curativo, inclusive com visitas domiciliares, e a avaliação de benefícios e custos que devem ser considerados no momento da escolha do tipo de curativo adequado, à natureza, à localização e ao tamanho da ferida ${ }^{2}$.

O hidrogel de carboximetilcelulose é um polímero natural utilizado no tratamento de feridas, classificado como curativo primário e de excelência para hidratação e manutenção de um ambiente úmido na ferida. Possuem a propriedade de absorver, desencrostar e desbridar o tecido necrótico e fibrótico. Encontram-se disponíveis sob diversas formas, que se baseiam em uma variedade de polímeros distintos ${ }^{13-15}$.

\section{Metodologia}

Trata-se de um estudo de intervenção terapêutica, prospectivo, do tipo ensaio clínico não controlado sobre o custo e a efetividade da carboximetilcelulose (CMC) a 2\% produzida numa farmácia universitária para o tratamento de feridas em pacientes com úlceras de perna. $\mathrm{O}$ estudo foi aprovado pelo Comitê de Ética em Pesquisa da Universidade Federal Fluminense com o $\mathrm{n}^{\mathrm{O}}$ 0154.0.258.000-08 e ocorreu no período de janeiro a outubro de 2010.

Foram admitidos no estudo 20 voluntários, segundo amostragem probabilística aleatória, dos pacientes que já se encontravam em acompanhamento no Ambulatório de Reparo Tecidual do hospital universitário no município de Niterói/Estado do Rio de Janeiro. Os critérios de inclusão para o estudo foram: voluntários com idade acima de 18 anos; com indicação para o uso da carboximetilcelulose (CMC) a 2\%; ausência de processo infeccioso na lesão; que apresentassem lesão crônica de perna em um ou ambos os membros inferiores; com condições cognitivas, a fim de seguir as orientações recomendadas durante o período do estudo. Esta pesquisa apresentou uma perda de 20\% do grupo amostral (quatro voluntários) por motivos que 
impediram sua continuidade, sendo eles: por ocorrência de infecção local e sistêmica sendo necessária a troca do tratamento; por solicitação do paciente que relatava frequentes queixas álgicas; por alteração do estado psíquico; por complicações de saúde que demandaram internação hospitalar e afastamento do estudo. Sendo assim, 16 voluntários foram acompanhados por 90 dias para a coleta de dados.

\section{$\mathrm{O}$ produto}

A CMC a 2\% foi produzida na Farmácia Universitária da Faculdade de Farmácia da Universidade Federal Fluminense, seguindo a formulação de um gel amorfo e não estéril. Sua composição foi de: 2\% de CMC, 0,1\% de metilparabeno, 20\% de propilenoglicol e 77,9\% de água purificada em frascos de $100 \mathrm{~g}$. A produção de um gel não estéril requer meios de controle de qualidade e efetividade do produto periodicamente. Dessa forma, a CMC a $2 \%$ passou por processamentos de Controle Físico-Químico e Controle Microbiológico. Todos os testes aplicados para o produto de estudo obtiveram resultados satisfatórios, não havendo impedimento para o seu uso nos voluntários do estudo.

\section{Avaliação da efetividade}

A avaliação da efetividade do gel de CMC a $2 \%$ foi realizada por meio de instrumento com dados clínicos, das mensurações das feridas realizadas a cada 15 dias, da fotografia digital e do decalque das lesões. Os decalques foram realizados a partir de folhas de acetato e transferidos para papel milimetrado, tendo como parâmetros a redução do tamanho das feridas e as alterações das características teciduais em quatro tempos: início do tratamento, 30 dias, 60 dias e 90 dias. A presença dos tecidos de epitelização, granulação, esfacelo e necrose foi avaliada por intermédio de uma escala ordinal validada para o estudo: $0=1$, $1-25 \%=2, \quad 26-40 \%=3,41-75 \%=4$ e $75-100 \%=5$.

\section{Avaliação dos custos}

Foram registrados os gastos dos materiais utilizados no procedimento de curativo no ambulatório e os gastos dos materiais entregues ao paciente para o curativo domiciliar - kit curativo. A perspectiva escolhida para o estudo foi a do Sistema Único de Saúde (SUS), como órgão comprador de serviços. Apenas os custos diretos cobertos pelo sistema público foram computados. Isto inclui os procedimentos cobertos pelas tabelas de reembolso de procedimentos do SUS tais como: ComprasNet, Sistema de Gerenciamento da Tabela de Procedimentos, Medicamentos e Órteses e Próteses e Materiais especiais do SUS (SIGTAP) e Banco de Preços do Ministério da Saúde. Desse modo, outros custos incorridos pelo SUS, como aqueles relacionados aos tratamentos de longo prazo, ao uso de medicamentos, aos serviços sociais, à administração e campanhas públicas não foram medidos no período de análise.

\section{Análise estatística}

Foram utilizadas as análises estatísticas descritiva e inferencial. A análise descritiva foi empregada nas variáveis de evolução das úlceras no período de 90 dias. Para análise estatística inferencial, foram usados os testes estatísticos de Friedman, Mann-Whitney e Kruskal-Wallis, com p-valor menor que 0,05 $(\rho<0,05)$ de nível de significância estatística, e a Correlação de Pearson para comparação das médias na redução de áreas e custo dos tratamentos analisados.

\section{Resultados e Discussão}

\section{Evolução das úlceras de perna}

Dos 16 voluntários atendidos no Ambulatório de Reparo Tecidual no período de estudo, com relação à evolução das úlceras de perna em uso da CMC a 2\%, observou-se que a redução das lesões foi maior nas primeiras semanas de tratamento, o que indica bom prognóstico com a terapêutica implementada e repercussão na cicatrização tecidual. As reduções de área nas lesões durante todas as semanas de acompanhamento apresentaram fortes correlações, uma vez que as reduções das úlceras de perna apresentaram um p-valor igual a 0,0317 e um fator de correlação igual a 0,5 conforme mostra a Tabela 1. É importante ressaltar que, dos 16 voluntários

TABELA 1: Evolução das úlceras de perna dos voluntários em uso de CMC a 2\% em 90 dias de tratamento. Niterói/RJ, 2010.

\begin{tabular}{cccc}
\hline $\begin{array}{c}\text { Volun- } \\
\text { tários }\end{array}$ & \multicolumn{2}{c}{ Tamanho da úlcera $\left(\mathbf{c m}^{2}\right)$} & \multicolumn{2}{c}{$\begin{array}{c}\text { Índice de Redu- } \\
\text { (nício } \\
\left(\mathbf{1}^{\circ} \text { dia }\right)\end{array}$} & $\begin{array}{c}\text { Final } \\
(90 \text { dias })\end{array}$ & $\begin{array}{c}\text { ça período } \\
\left.\text { de } 90 \text { dias }(\%)^{*}\right)\end{array}$ \\
\hline 1 & 19 & 13 & 68,4 \\
2 & 296 & 215 & 72,6 \\
3 & 43 & 33 & 76,7 \\
4 & 6 & 4 & 66,6 \\
5 & 4,25 & 0 & 100,0 \\
7 & 4 & 0 & 100,0 \\
8 & 34 & 18 & 52,9 \\
9 & 2 & 1 & 50,0 \\
10 & 5 & 0 & 100,0 \\
11 & 30,25 & 0 & 100,0 \\
14 & 53 & 42 & 79,2 \\
16 & 197 & 160 & 81,2 \\
17 & 46 & 7 & 15,2 \\
18 & 213 & 170 & 79,8 \\
19 & 138,5 & 170 & - \\
20 & 18 & 6 & 33,3 \\
\hline
\end{tabular}

${ }^{(*)} \mathrm{p}<0,05$ e $Q=0,5$, onde: $\mathrm{p}=\mathrm{p}$-valor e $\mathrm{Q}=$ coeficiente de correlação de Pearson. 
estudados, 4(25\%) chegaram à cicatrização tecidual total, enquanto que os outros 12(75\%) apresentaram redução da úlcera, mas não chegaram à cicatrização completa das lesões, permanecendo com área lesional até a $12^{\mathrm{a}}$ semana de tratamento.

Ao se comparar o índice de redução das lesões no início e no término do acompanhamento clínico (após 90 dias), percebeu-se que 14(93,75\%) voluntários do estudo tiveram a área lesional reduzida com uso de carboximetilcelulose a 2\%, que variou de 20,19\% a $100 \%$ da área total. A média de redução dos 16 voluntários do estudo foi de 50,07\% $\pm 37,7$ - de acordo com o apresentado na Tabela 1. Para alguns estudiosos ${ }^{16-19}$, o tempo necessário para a cicatrização de uma úlcera de perna depende de uma série de fatores físicos, incluindo a área inicial da úlcera, delimitada pela borda da ferida, a profundidade inicial da lesão, a localização das úlceras e a taxa de migração de fibroblastos e queratinócitos para o leito lesional.

Detectou-se que, nos voluntários que não tiveram redução de fibrina, a maioria permaneceu com suas lesões abertas até a $12^{\mathrm{a}}$ semana de tratamento. Tal fato reforça a afirmação dos estudos cuja identificação da presença de fibrina em $50 \%$ ou mais da superfície da ferida sugere um prognóstico desfavorável em sua cicatrização. Dificilmente, pacientes com evolução insatisfatória do leito terão suas lesões cicatrizadas, haja vista que a presença de tecidos inviáveis, além de favorecer infecções, não permite a formação de tecido de granulação e adequada reepitelização ${ }^{20,21}$. É razoável supor que a evolução satisfatória observada no estudo pôde ser atribuída ao CMC a 2\%, uma vez que o gel tem como função a redução da fibrina, por meio do desbridamento autolítico, além de promover a epitelização e a manutenção do leito úmido da ferida ${ }^{5,7,8,22}$.

\section{Avaliação econômica da carboximetilcelu- lose $2 \%$}

A fim de avaliar melhor o uso dA CMC a 2\%, é apresentada a redução dos custos referentes aos 90 dias de acompanhamento das úlceras de perna nos voluntários, de acordo com o tamanho da área categorizada pelo estudo. São elas: área A [acima de $100 \mathrm{~cm}^{2}$ ] e área B [abaixo de $100 \mathrm{~cm}^{2}$ ]. Notou-se que a redução dos custos foi de $\mathrm{R} \$ 14,66$ e $\mathrm{R} \$ 43,86$ $(\mathrm{p}<0,0001)$, respectivamente. Observa-se que, no grupo A, houve um aumento no segundo mês, que sugere ser o processo de adaptação do produto terapêutico ao paciente, conforme ilustrado na Figura 1.

No que se refere ao custo do tratamento com recursos materiais, ao se analisar o preço médio dos produtos utilizados para o tratamento das feridas com CMC a 2\% manipulado na farmácia universitária durante um mês de tratamento, chegou-se a uma média de custo mensal de $\mathrm{R} \$ 70,75$ para o grupo de voluntários da área B e $\mathrm{R} \$ 112,85$ para os perten-

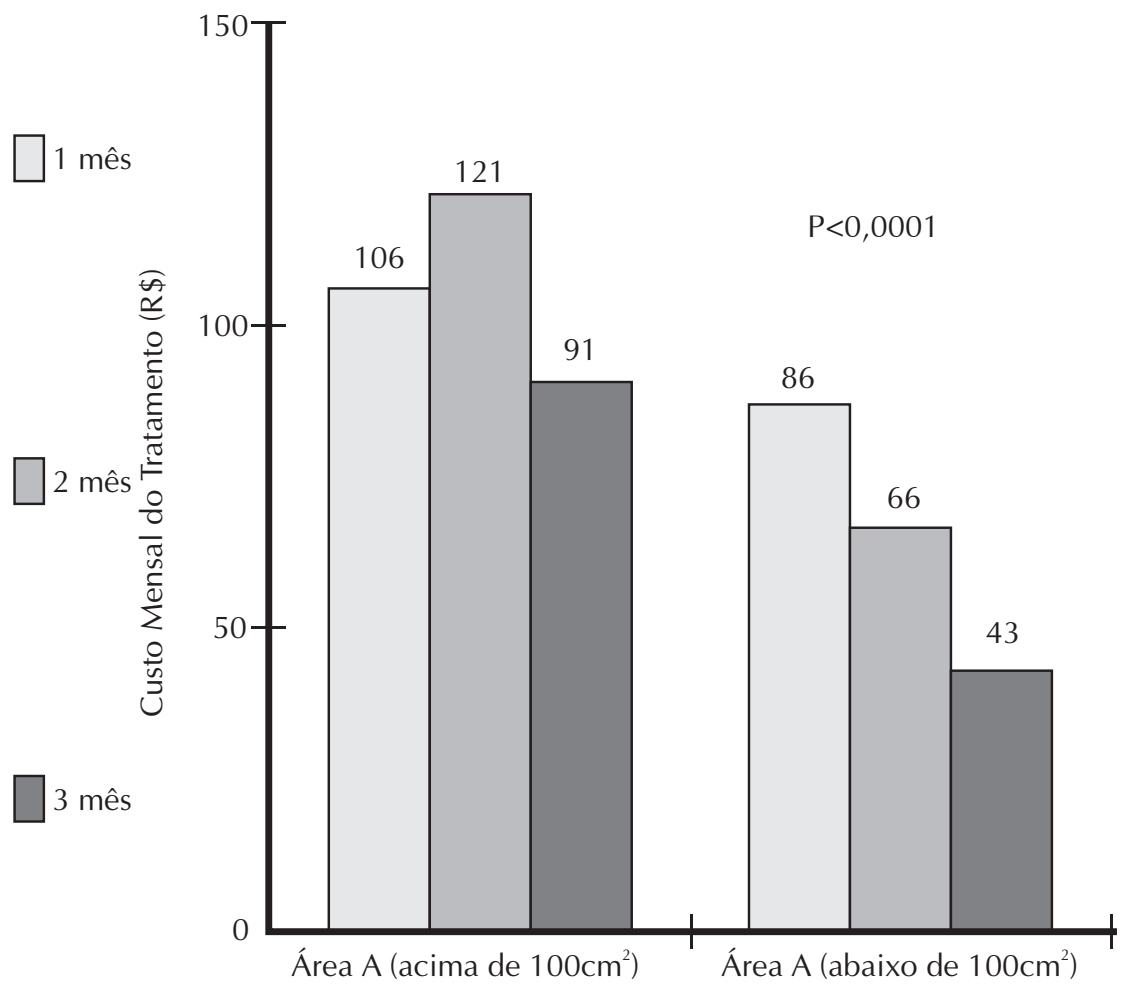

FIGURA 1: Comparação do custo mensal do tratamento com CMC a $2 \%$ segundo o tamanho da área lesional dos voluntários atendidos durante os 90 dias de tratamento. Niterói/RJ, 2010. 
centes à área $\mathrm{A}$, de acordo com o apresentado na Figura 2. Tal afirmação já foi comprovada por estudo segundo o qual o tempo total para a cicatrização é um dos fatores que mais influenciavam os custos para o tratamento ${ }^{23}$.
Quando se efetuou a relação proporcional da quantidade de gel dos blisters nas amostras disponíveis no mercado, com a carboximetilcelulose a $2 \%$ de 100 gramas, observou-se que os valores correspondentes às áreas de tamanho da lesão variaram

CMC 2\% $100 \mathrm{~g}$

gel 3

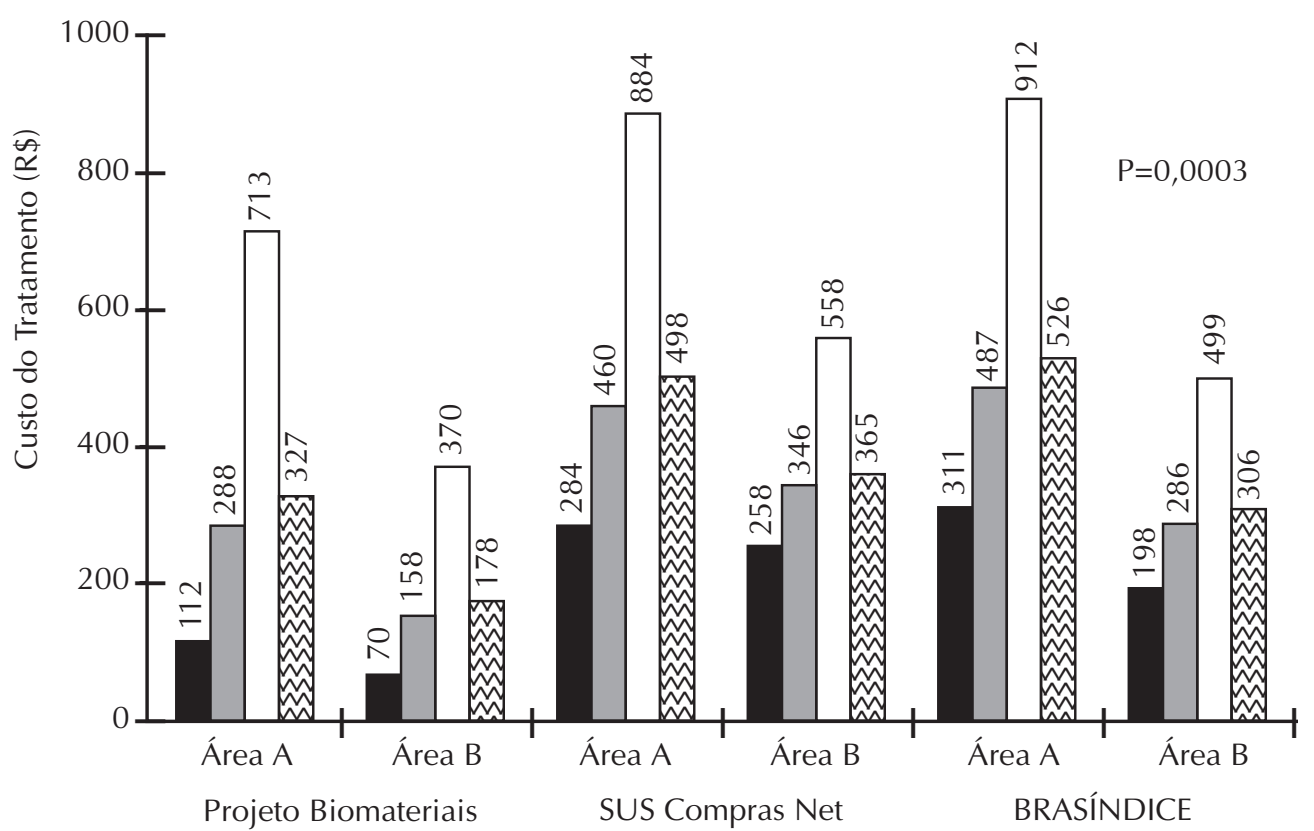

FIGURA 2: Comparação do custo do tratamento das úlceras de perna com CMC a 2\% de $100 \mathrm{~g}$ e géis proporcionais a $100 \mathrm{~g}$ nas três esferas do Projeto de Pesquisa. Niterói/RJ, 2010.

de acordo com a referência-padrão de 100 gramas da carboximetilcelulose a $2 \%(p=0,0003)$. Os achados mostraram que, ainda com a proporção dos géis industrializados para a quantidade de 100 gramas, como a carboximetilcelulose a $2 \%$, os valores permanecem acima do valor real, independente do tamanho da área afetada, como mostrado na Figura 2. Um estudo demonstrou que o custo semanal associado às úlceras de longa duração é mais do que o dobro do custo relacionado ao tratamento das lesões de curta duração, independentemente do tamanho da úlcera, o que ocorreu devido ao aumento das despesas com pessoal, como resultado direto de mais frequentes e longas trocas de curativos e de um grande período para a cura ${ }^{5,7,24}$.

\section{Conclusão}

O estudo revelou que o tratamento das úlceras de perna com a CMC a 2\% apresentou uma significativa redução do edema, da dor, da maceração e, ainda, uma evolução satisfatória do leito da ferida, uma vez que promoveu o aumento do tecido de granulação até sua epitelização.

Conclui-se que o uso do gel de carboximetilcelulose a 2\% magistral contribuiu não só para a aceleração do processo cicatricial, mas também para a redução dos custos do tratamento quando comparados com outros géis disponíveis no mercado nacional.

A carboximetilcelulose a $2 \%$, manipulada magistralmente numa farmácia universitária, representou uma alternativa para o tratamento de úlceras de perna no SUS, uma vez que promoveu melhora nas taxas de cicatrização, diminuição do tempo de tratamento e baixo custo, trazendo menores gastos para os serviços de saúde e órgãos de financiamento.

O considerável crescimento tecnológico aumenta a oferta de produtos para curativo no mercado; entretanto, é preciso avaliar os custos e os benefícios destes produtos e, principalmente, o potencial para desenvolvimento nacional, bem como o fornecimento de uma assistência sistematizada com base nas melhores evidências científicas.

\section{REFERÊNCIAS}

1.Sevegnani PO, Burim SFF, Filus WA. Custos diretos de curativos em úlcera por pressão: estudo de caso. Bol Enferm. 2007; 1:46-65.

2.Franco D, Gonçalves LF. Feridas cutâneas: a escolha do curativo adequado. Rev Col Bras Cir. 2008; 35:203-6. 3.Ministério da Saúde (Br). Diretrizes metodológicas: 
estudos de avaliação econômica de tecnologias em saúde. Brasília (DF): Ministério da Saúde; 2009.

4.Ministério da Saúde (Br). Política nacional de gestão de tecnologias em saúde. Brasília (DF): Ministério da Saúde; 2010.

5.Carmo SS, Castro CD, Rios VS, Sarquis MGA. Atualidades na assistência de enfermagem a portadores de úlcera venosa. Rev eletrônica enferm. 2007; 9:506-17. 6.Abbade LPF. Abordagem do paciente portador de úlcera venosa. In: Malagutti W, Kakibara CT. Curativos, estomias e dermatologia: uma abordagem multiprofissio-

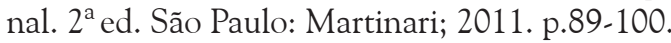

7.Barbosa JAG, Campos LMN. Diretrizes para o tratamento da úlcera venosa. Enferm glob. 2010; 20:1-13.

8.Scottish Intercollegiate Guidelines Network (UK). Management of chronic venous leg ulcers. A national clinical guideline [site de internet] 2010. [citado em 28 jun 2013] Disponível em: http://www.sign.ac.uk/pdf/ sign120.pdf

9.Borges EL. Feridas - úlceras de dos membros inferiores. Rio de Janeiro: Guanabara Koogan; 2011.

10.Scemons D, Elston D. Nurse to nurse: cuidados com feridas em enfermagem. São Paulo: Mc Graw-Hill; 2011. 11.Silva FAA, Freitas CHA, Jorge MSB, Moreira TMM, Alcântara MCM. Enfermagem em estomaterapia: cuidados clínicos ao portador de úlcera venosa. Rev Bras Enferm. 2009; 62:889-93.

12.Sant'ana SMS, Bachion MM, Santos QR, Nunes CAB, Malaquias SG, Oliveira BGRB. Úlceras venosas: caracterização clínica e tratamento em usuários atendidos em rede ambulatorial. Rev Bras Enf. 2012; 65:637-44. 13.Medeiros WS, Oliveira BGRB, Granjeiro JM. Biomateriais e o corpo. In: Figueiredo NMA, Machado WCA. Corpo e saúde: condutas clínicas do cuidar. Rio de Janeiro: Águia Dourada; 2009. p.89-97.

14.Rodrigues LM, Souza CJ. Assistência de enfermagem em lesões de pele e úlceras por pressão. In: Souza CJ. Manual de rotina em enfermagem intensiva. Rio de Janeiro: Guanabara Koogan; 2010. p.247-302.

15.Iponema E, Costa MM. Úlceras vasculogênicas. In: Silva CRL, Figueiredo NMA, Meireles IB. Feridas: fundamentos e atualizações em enfermagem. São Caetano do Sul: Yendis Editora; 2007. p.381-96.
16. Rabeh SAN, Gonçalves MBB, Caliri MHL, Nogueira PC, Miyazak MY. Construção e validação de um módulo educativo virtual para terapia tópica em feridas crônicas. Rev enferm UERJ. 2012; 20:603-8.

17.Tennvall GR, Hjelmgren J, Oien R. The cost of treating hard-to-heal venous leg ulcers: results from a Swedish survey [site de Internet] 2006. [citado em 28 jun 2015] Disponível em: http://www.worldwidewounds. com/2006/november/Tennvall/Cost-of-treating-hardto-heal-venous-leg-ulcers.html

18.Abreu AM, Oliveira BGRB, Manarte JJ. Treatment of venous ulcers with an unna boot: a case study. Online Braz J Nurs. 2013; 12(1):198-208.

19.Stephen-Haynes J. Leg ulceration and wound bed preparation: towards a more holistic framework [site de Internet] 2007. [citado em 23 nov 2014] Disponível em: http://www.worldwidewounds.com/2007/october/ Stephenhaynes

20.Dealey C. Cuidando de feridas: um guia para enfermeiras. $3^{\mathrm{a}}$ ed. São Paulo: Atheneu; 2008.

21.Agency for Healthcare Research and Quality (US). Chronic Venous Ulcers: A Comparative Effectiveness Review of Treatment Modalities [site de Internet] 2012. [citado em 28 jun 2015] Disponível em: http:// effectivehealthcare.ahrq.gov/ehc/products/367/995/ CVU_AmendedProtocol_20120913.pdf

22.Morley S. Management of chronic venous leg ulcers: a national clinical guideline. Scottish Intercollegiate Guidelines Network [site de Internet] 2010. [citado em 28 jun 2015] Disponível em: http://www.sign.ac.uk/pdf/sign120.pdf 23.Pálsdóttir G. Chronic leg ulcers in Iceland: Prevalence, aetiology and management [site de Internet] 2009. [citado em 28 jun 2015] Disponível em: http://skemman. is/stream/get/1946/2296/7329/1/

24.Australian Wound Management Association Inc (AU). Australian and New Zealand Clinical Practice Guideline for Prevention and Management of Venous Leg Ulcers. New Zealand (Au): Cambridge Publishing; 2011. p.30-61. 24.Australian Wound Management Association Inc (AU). Australian and New Zealand Clinical Practice Guideline for Prevention and Management of Venous Leg Ulcers. New Zealand (Au): Cambridge Publishing; 2011. p.30-61. 